\title{
Influência da distância geográfica na riqueza e composição de espécies arbóreas em uma Floresta Ombrófila Densa na Amazônia Oriental
}

\author{
Influence of geographical distance in richness and composition of tree species \\ in a tropical rain forest in eastern Amazonia
}

Darley C. Leal Matos ${ }^{1,2}$, Leandro Valle Ferreira ${ }^{2} \&$ Rafael de Paiva Salomão ${ }^{1}$

\begin{abstract}
Resumo
Duas teorias em ecologia explicam a distribuição de espécies em florestas tropicais: a Teoria Neutra e a Teoria do Nicho. Na primeira a distribuição de espécies está relacionada a processos como dispersão, enquanto a segunda prediz que é devido às interações competitivas entre as espécies. Estudos demonstram que a similaridade de espécies de uma comunidade vegetal diminui com a distância geográfica nas regiões tropicais. O objetivo deste estudo foi identificar se a distância influencia nos padrões de riqueza e composição de espécies de árvores dentro e entre seis platôs em uma floresta ombrófila densa na Floresta Nacional Sacará-Taquera, Pará, Brasil. Esses platôs são explorados para a extração da bauxita, onde a cobertura florestal é totalmente removida. O número de parcelas $(10 \times 250 \mathrm{~m})$ analisadas nesse estudo, em cada platô variou de 18 a 22 . Houve uma correlação negativa da similaridade de espécies em relação à distância, tanto dentro como entre os platôs. Não houve correlação da riqueza de espécies em relação à distância, dentro e entre os platôs, com exceção do platô Aramã. Os resultados desse estudo corroboram a Teoria Neutra e têm grandes implicações para a conservação.

Palavras-chave: complementaridade, Floresta tropical, Platô, Teoria Neutra, Teoria do Nicho.
\end{abstract}

\begin{abstract}
Two theories in ecology explain species distribution in tropical forests: the Neutral Theory and Niche Theory. The first predicts the species distribution is related to processes such as dispersion, and the second predicts that species distribution is related to competitive interactions between species. Studies have shown that the species similarity in a plant community decreases according to the geographical distance in the tropics. The aim of this study was to assess the influence of the distance on tree species richness and composition within and among six plateaus in a tropical rain forest at the Sacará-Taquera National Forest, Pará, Brazil. These plateaus are exploited for the bauxite extraction where the forest cover is completely removed. In this study, the number of plots used in each plateau ranged from 18 to 22 . There was a negative correlation of the species similarity to the distance, whithin and among the plateaus. There was no correlation of species richness to distance (whithin and among the plateaus), except for the plateau Aramã. The results of this study support the Neutral Theory and have important implications for the conservation as well.
\end{abstract}

Key words: complementarity, Tropical forest, Plateau, Neutral Theory, Niche Theory.

\section{Introdução}

A distribuição das espécies no espaço é base para o desenvolvimento de teorias em ecologia de comunidades, como a Teoria Neutra de Hubbell (2001) e a Teoria do Nicho (Macarthur \& Levins 1964; Wight 2002; Kneitel \& Chase 2004) que propõem os mecanismos condutores da abundância e distribuição de espécies em comunidades.

A Teoria Neutra assume que todos os indivíduos de uma comunidade são funcionalmente equivalentes, apresentam a mesma probabilidade de migrar, reproduzir e morrer, e que a abundância de espécies é dependente do acaso e não da superioridade competitiva de um indivíduo. A

\footnotetext{
'Museu Paraense Emílio Goeldi, Coord. Botânica, Av. Perimetral 1901, Terra Firme, 66077-530, Belém, PA, Brasil.

${ }^{2}$ Autora para correspondência: dcleal_eco@yahoo.com.br
} 
coexistência das espécies em diferentes escalas é resultado de um equilíbrio entre imigração e extinção em escala local e entre as taxas de especiação e extinção em escala regional. A teoria prediz que a similaridade florística diminui com o aumento da distância geográfica entre locais, independentemente das diferenças ambientais entre eles, mas sim por causa da limitação de dispersão no espaço. Assim, os mecanismos que geram diferenças nos padrões de composição das espécies estão ligados à capacidade de dispersão dos indivíduos (Hubbell 2001; 2006).

As predições da teoria neutra são opostas à teoria tradicional de assembléias de nichos a qual assume que as espécies diferem entre si exibindo certa diferenciação de nicho, de forma que os recursos são utilizados diferencialmente por cada espécie. Para coexistirem em uma comunidade, as espécies apresentam trade-offs, ou seja, a especialização na obtenção de um determinado tipo de recurso normalmente vem acompanhada de uma diminuição na eficiência com relação a outro (Mikkelson 2005; Condit et al. 2006; Giacomini 2007). Dessa forma, o padrão de composição das espécies deve ser determinado por características ambientais que relacionam as espécies capazes de se estabelecer em certo local.

Diversos estudos mostram que a distância geográfica influencia na distribuição de plantas em regiões tropicais (Scudeller et al. 2001; Condit et al. 2002; Ruokolainen \& Tuomisto 2002; Carneiro \& Valeriano 2003), e atribuem a variação de riqueza e composição de espécies entre locais basicamente à limitação de dispersão. Esses estudos corroboram a Teoria Neutra devido à similaridade de espécies diminuir com o aumento da distância entre parcelas.

Muitos outros estudos mostram que a variação na riqueza e composição de espécies está associada à distribuição descontínua de fatores ambientais, tais como, tipos de solo, topografia, altitude e clima, sendo esta variação dependente da escala (Tuomisto et al. 1995; Tuomisto \& Poulsen 1996; Ruokolainen \& Tuomisto 2002). Com o aumento da distância há um decréscimo na similaridade ambiental entre locais, formando gradientes de condições ambientais (Nekola \& White 1999). Nestes casos as comunidades são estruturadas pelo nicho, e a distribuição das espécies de plantas está relacionada à distribuição heterogênea dos fatores ambientais no espaço (Pyke et al. 2001; Zumquim 2007).
As florestas tropicais, especialmente a Amazônia, são caracterizadas pela alta riqueza de espécies e baixa similaridade de espécies entre locais, tanto em escala local como em escalas regionais (Nekola \& White 1999; Tuomisto et al. 2003), sendo isto associado ao pequeno número de espécies comuns entre locais. A diferença na riqueza e composição de espécies entre locais é atribuída à diversidade beta, resultante da heterogeneidade de habitats e da limitação de dispersão das espécies (Ter Steege et al. 2000; Tuomisto et al. 2003; Costa et al. 2005).

A variação da riqueza e composição de espécies em comunidades vegetais em diferentes escalas espaciais também é importante para explicar porque as regiões tropicais são caracterizadas pela alta riqueza e baixa similaridade de espécies (Mazancourt 2001).

Na Floresta Nacional (FLONA) de SacaráTaquera na Amazônia, município de Oriximiná no estado do Pará, existem diversos platôs situados a diferentes distâncias, recobertos por floresta ombrófila densa primária (Veloso et al. 1991). Nos platôs a variação na riqueza e composição de espécies de plantas pode ser testada em escala local (dentro dos platôs) e regional (entre os platôs). Ferreira et al. 2011 encontrou uma correlação negativa da similaridade com a distância em 179 parcelas de $10 \times 250 \mathrm{~m}$ instaladas no Platô Bela Cruz na Flora Sacará-Taquera, demonstrando que mesmo em nível local o aumento da distância limita a dispersão das espécies diminuindo a similaridade entre parcelas próximas. Assim, este estudo tem como objetivo identificar se a distância geográfica influencia nos padrões de riqueza e composição de espécies de árvores dentro e entre platôs em uma Floresta Ombrófila Densa na Amazônia Oriental.

Neste estudo, foram avaliadas as seguintes questões: a) a distância influencia no padrão de distribuição das espécies arbóreas entre e dentro dos platôs? b) a similaridade florística aumenta ou diminuiu à medida que a distância aumenta entre as parcelas; c) existe algum padrão espacial da riqueza de espécies nos platôs?

\section{Material e Métodos}

Área de estudo

Este estudo foi realizado na Floresta Nacional (FLONA) Sacará-Taquera $\left(01^{\circ} 21^{\prime}-6^{\circ} 22^{\prime} \mathrm{W}\right)$, localizada no município de Oriximiná, Pará. Nesta FLONA está inserido o projeto de mineração de bauxita da Mineração Rio do Norte (MRN) (Fig. 1). 


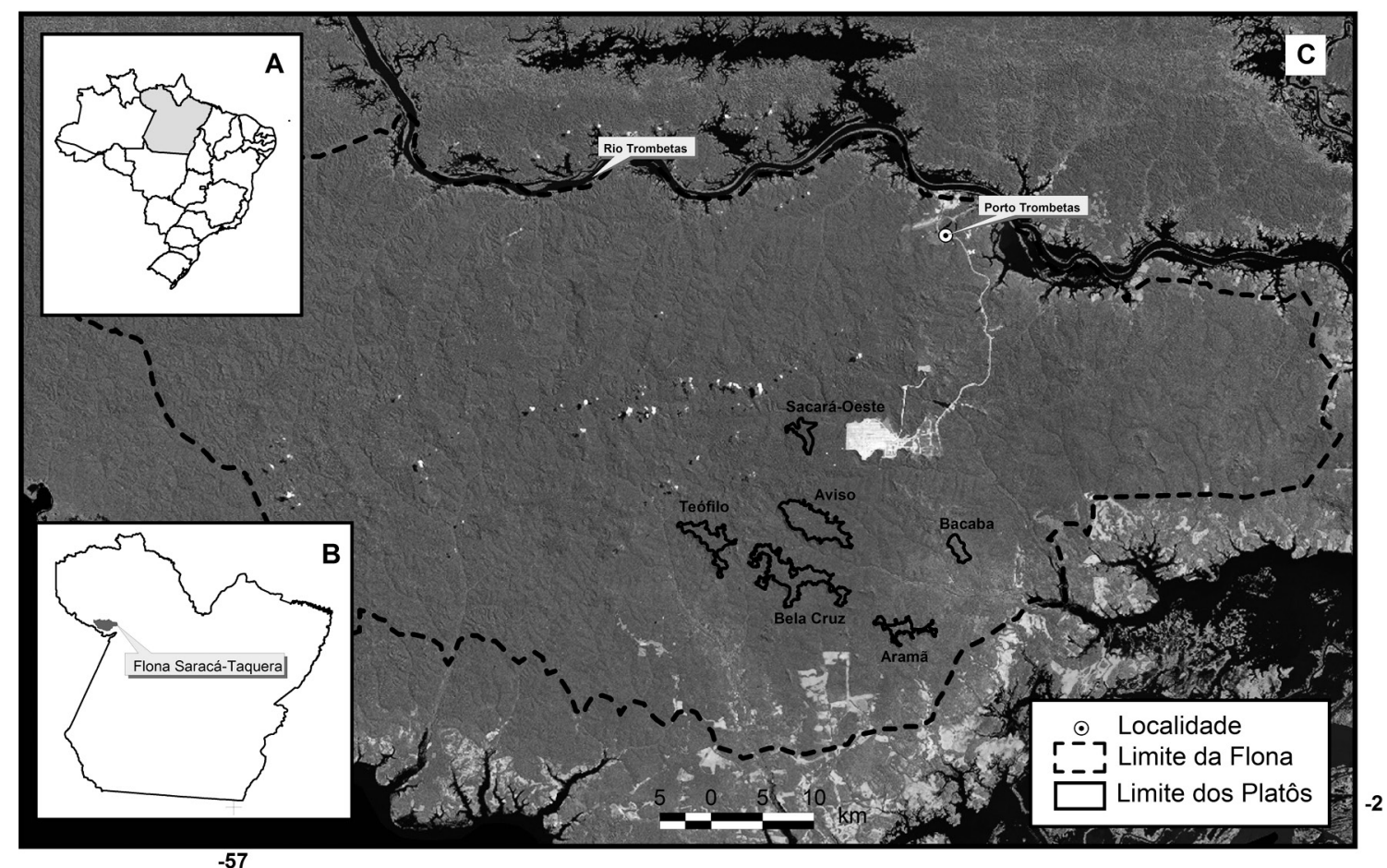

Figura 1 - Localização do estado do Pará (A), da Floresta Nacional Sacará-Taquera no município de Oriximiná (B), e a localização dos seis platôs utilizados neste estudo (Fonte: Mineração Rio do Norte, 2005).

Figure 1 - Location of Pará State (A), the National Forest Sacará-Taquera in the city of Oriximiná (B), and the location of the six plateaus used in this study (Image from Mineração Rio do Norte, 2005)..

O processo de exploração da bauxita ocorre em platôs bem definidos, recobertos pela Floresta Ombrófila Densa (RadamBrasil 1976). Antes da exploração ocorre um inventário botânico dos platôs, como subsídio para a obtenção da licença de desmatamento para extração do minério. $\mathrm{O}$ inventário botânico possibilita o aproveitamento da madeira comercial e subsidia as ações de recuperação florestal das áreas desmatadas (Salomão et al. 2007).

Nos platôs inventariados neste estudo, a variação dos fatores abióticos é relativamente pequena. O clima da região é o Af segundo a classificação Köppen, caracterizado como Tropical Úmido, que apresenta precipitação pluviométrica média anual variando entre $2.500 \mathrm{~mm}$ e 3.000 $\mathrm{mm}$. Predominam na área o Latossolo Amarelo distrófico, textura muito argilosa e o Latossolo Amarelo distrófico textura argilosa, que são solos profundos e normalmente sem problemas de drenagem, sob floresta densa de relevo tabular de topo plano (RadamBrasil 1976). A elevação varia de 50 a 120 m, e a altitude de 150 a $200 \mathrm{~m}$ (Salomão et al. 2007). A distância entre os platôs varia de 5,3 (entre o platô Aviso e Bela Cruz) a 23,7 km (entre o platô Bacaba e Teófilo).

\section{Coleta de dados}

Neste estudo foi utilizado um banco de dados pré-existente de seis inventários florestais realizados nos platôs Aramã, Aviso, Bacaba, Bela Cruz, Saracáoeste e Teófilo (Salomão et al. 2007). Em cada platô foram distribuídas sistematicamente parcelas de 10 $\times 250$ metros cobrindo toda área do platô.

Dentro de cada parcela todos os indivíduos (árvores, palmeiras e cipós) com DAP (diâmetro a $1,30 \mathrm{~m}$ do solo) $\geq 10 \mathrm{~cm}$ foram medidos e identificados, com coleta de material botânico para confirmação de identificação e herborização no Herbário João Murça Pires do MCT/Museu Paraense Emílio Goeldi (MG).

Para este estudo foi utilizado um critério de escolha das parcelas baseado na distância entre as parcelas dentro dos platôs, sendo escolhidos grupos de parcelas localizadas nas extremidades e no centro de cada platô (Fig. 2). O número de parcelas analisadas em cada platô variou de 18 a 22, totalizando 123 parcelas. 


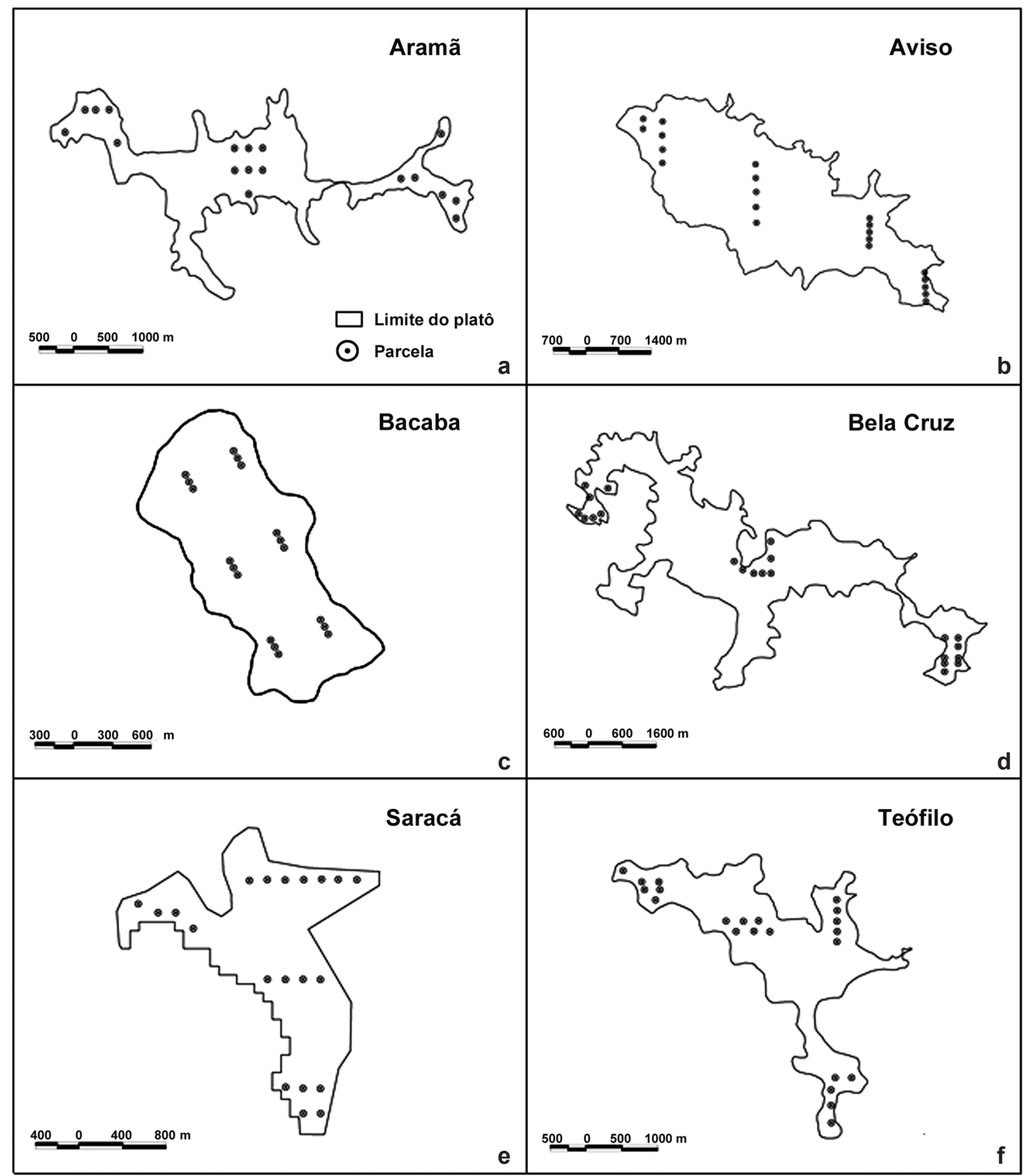

Figura 2 - Distribuição das parcelas analisadas no platô Aramã, Aviso, Bacaba, Bela Cruz, Sacará-oeste e Teófilo para este estudo. Figure 2 - Distribution of plots analyzed in the plateau Aramã, Aviso, Bacaba, Bela Cruz, Sacará-oeste and Teófilo for this study.

Análise de dados

Os dados de diversidade e composição de espécies foram obtidos no programa Mata Nativa 2 (Cientec 2006). O índice de diversidade utilizado foi o de Shannon (Magurran 1988), e a similaridade de espécies dentro e entre platôs foi calculada pelo índice de similaridade de Sorensen (Krebs 1999). Uma matriz de similaridade florística das parcelas dentro e entre os platôs foi gerada no programa Mata Nativa (Cientec 2006), utilizando o índice de similaridade de Sorensen. 
Uma matriz de riqueza de espécies foi obtida utilizando a distância euclidiana para os valores de riqueza das parcelas dentro e entre os platôs, usando o programa PCORD 4 (Mccune \& Mefford 1999).

A área dos platôs foi obtida através do mapa de desmatamento produzido pela Mineração Rio do Norte (MRN), Projeto Trombetas, e analisada no programa Arcview 3.3 (Esri 2006). As distâncias das parcelas dentro e entre os platôs foram calculadas com a extensão "Distance Matrix of Point Features", usando o programa Arcview 3.3 (Esri 2006).

As curvas cumulativas de espécies e dos estimadores de riqueza foram feitas no programa Estimates 8 (Statiscal Estimation of species Richness and Shared Species form Samples) (Cowell \& Coddington 1996). Foi utilizado o estimador não paramétrico Jackknife de $1^{\text {a }}$ ordem, pois este possibilita estimar o número total de espécies utilizando o número de espécies que ocorrem em apenas uma amostra (Colwell 1997; Krebs 1999), ou seja, estima a riqueza total somando a riqueza observada (número de espécies coletado) a um parâmetro calculado a partir do número de espécies raras (aquelas que ocorreram em uma amostra).

Diagramas de dominância (Whittaker 1965) foram feitos ordenando as espécies, das mais comuns para as mais raras. As abundâncias das espécies foram transformadas utilizando $\log 10(\mathrm{n}+1)$ (Magurran 2004).

Para testar a correlação das matrizes de similaridade e riqueza de espécies em relação à distância dentro e entre os platôs foi utilizado o teste de Mantel (Manly 1994), sendo a significância das correlações testada através do teste de Monte Carlo com 1.000 permutações aleatórias, usando o programa PCORD 4 (Mccune \& Mefford 1999).

\section{Resultados}

Riqueza e diversidade de espécies entre platôs

Foram identificadas 929 espécies nas 123 parcelas amostradas e analisadas nos seis platôs. O número de espécies variou de 259 no platô Aramã a 401 espécies no platô Aviso. O índice de diversidade variou de 4,2 no platô Bacaba, a 5,02 no platô Saracá-Oeste (Tab. 1).

As curvas cumulativas de espécies não exibiram tendência de assíntota nos seis platôs analisados. Contudo, a proporção entre o número de espécies observadas em relação às obtidas pelo estimador Jackknife 1 variou de $61,9 \%$ a $79,2 \%$, demonstrando que o número de parcelas usadas neste estudo conseguiu abranger mais de $50 \%$ da riqueza estimada (Fig. 3 e Tab. 2).

\section{Distribuição de abundância e de freqüência}

Em escala local, a distribuição de abundância das espécies nos seis platôs mostrou que poucas espécies são abundantes, sendo a maioria das espécies representadas por poucos indivíduos (Fig. 4).

Em escala regional, do total de 929 espécies encontradas nos seis platôs, 753 ( $81 \%$ do total) ocorreram em menos de $10 \%$ do total de parcelas, sendo desta forma consideradas espécies de baixa freqüência (Fig. 5).

Tabela 1 - Área total, número de espécies e índice de diversidade $\left(\mathrm{H}^{\prime}\right)$ nos seis platôs analisados neste estudo. Table 1 - Total area, species number and diversity index ( $\left.\mathrm{H}^{\prime}\right)$ in the six plateaus analyzed in this study.

\begin{tabular}{lccc}
\hline Nome do platô & $\begin{array}{c}\text { Área total } \\
\text { (hectares) }\end{array}$ & $\begin{array}{c}\mathbf{N}^{\mathbf{0}} \mathbf{d e} \\
\text { espécies }\end{array}$ & $\begin{array}{c}\text { H' } \\
\text { (Índice de } \\
\text { Shannon) }\end{array}$ \\
\hline Aramã & 416 & 259 & 4,59 \\
Aviso & 1436 & 401 & 5,01 \\
Bacaba & 284 & 277 & 4,2 \\
Bela Cruz & 1601 & 379 & 4,84 \\
Saracá-oeste & 364 & 320 & 5,02 \\
Teófilo & 784 & 325 & 4,74 \\
\hline
\end{tabular}

Tabela 2 - Número estimado de espécies, e a proporção da riqueza observada com a riqueza estimada por Jackknife 1 para os seis platôs analisados neste estudo. Table 2 - Estimated number of species and the proportion of richness observed with richness estimated by Jackknife 1 to six plateaus analyzed in this study.

\begin{tabular}{lccc}
\hline Platôs & $\begin{array}{c}\text { Riqueza total } \\
\text { nas parcelas } \\
\text { analisadas }\end{array}$ & $\begin{array}{c}\mathbf{N}^{\mathbf{0}} \text { de espécies } \\
\text { estimadas } \\
\text { Jackknife 1 }\end{array}$ & $\begin{array}{c}\text { \% de } \\
\text { espécies }\end{array}$ \\
\hline Aramã & 259 & 327 & 79,20 \\
Aviso & 401 & 516 & 77,71 \\
Bacaba & 277 & 382 & 72,51 \\
Bela Cruz & 379 & 508 & 74,61 \\
Saracá-oeste & 320 & 413 & 77,48 \\
Teófilo & 325 & 525 & 61,90 \\
\hline
\end{tabular}


Análise de autocorrelação espacial

Houve uma correlação negativa entre a similaridade de espécies e a distância geográfica entre as parcelas dentro dos seis platôs. Assim como ocorreu entre as parcelas dentro de cada platô, houve também correlação significativa negativa entre a similaridade florística e a distância geográfica entre os platôs (Tab. 3).

Houve uma correlação negativa significativa entre a riqueza e a distância entre as parcelas do platô Aramã. Nos demais platôs e entre eles não houve correlação (Tab. 3).

\section{Discussão}

A correlação negativa da similaridade de espécies em relação à distância em escala local (dentro) e regional (entre) nos platôs analisados neste estudo corrobora com a Teoria Neutra de Hubbell (2001), que prediz que a similaridade de espécies diminui com o aumento da distância entre locais, ou seja, quanto mais distantes as parcelas mais distintas elas são em nível de composição florística, e isto é resultado de limitações na dispersão (Hubbell 2001; Condit et al. 2002; Matos, 2009).

A dispersão das espécies é influenciada pela distância e escala com que os organismos utilizam a paisagem e pela capacidade de dispersão de cada espécie (Kalkhoven et al. 1993). Parece claro que, quanto menor for à capacidade de dispersão das espécies, maior será a influência da distância em variações na composição de espécies em escalas locais e regionais (Hubbell 1999), porque há um decréscimo nas trocas de indivíduos entre locais.

Tabela 3 - Índice de correlação e o valor da significância, da similaridade de espécies e da riqueza em relação à distância dentro e entre parcelas dos platôs analisados. Table 3 - Correlation coefficient and significance value, of the species similarity and richness with distance between and inside plots the plateaus analyzed.

\begin{tabular}{lcccc}
\hline & \multicolumn{2}{c}{ Similaridade } & \multicolumn{2}{c}{ Riqueza } \\
\hline Platô & $\boldsymbol{r}$ & $\boldsymbol{p}$ & $\boldsymbol{r}$ & $\boldsymbol{p}$ \\
\hline Aramã & $-0,57$ & 0,0001 & $-0,21$ & 0,013 \\
Aviso & $-0,28$ & 0,0001 & $-0,042$ & 0,563 \\
Bacaba & $-0,47$ & 0,0001 & $-0,164$ & 0,056 \\
Bela Cruz & $-0,51$ & 0,0001 & $-0,073$ & 0,294 \\
Saracá oeste & $-0,46$ & 0,0001 & $-0,074$ & 0,335 \\
Teófilo & $-0,39$ & 0,0001 & $-0,046$ & 0,5 \\
Entre platôs & $-0,49$ & 0,0001 & $-0,178$ & 0,625 \\
\hline & & & &
\end{tabular}

Organismos dotados de maior habilidade na dispersão tendem a contribuir para uma maior distribuição de espécies no ambiente, podendo aumentar a similaridade de espécies entre locais (Bell 2001).

Uma das explicações para a correlação negativa da similaridade de espécies em relação à distância, tanto dentro como entre os platôs, está provavelmente associada ao grande número de espécies raras, com baixa densidade e freqüência nos platôs analisados neste estudo, sendo este um padrão comumente encontrado nas florestas da Amazônia (Oliveira et al. 2008; Ferreira et al. 2011).

A Teoria Neutra prediz que haverá um declínio (quantitativamente estimável) na similaridade da comunidade ao longo do espaço, porque espécies se extinguirão e ocasionalmente serão substituídas por imigrantes de outras espécies, e isto depende da taxa de dispersão das espécies de fora da comunidade e nas restrições da dispersão dos organismos. Esse mecanismo explicaria o grande número de espécies raras em florestas tropicais, e, como estas têm grande propensão à extinção local, são substituídas mais lentamente na comunidade do que espécies comuns (Hubbell 2001; Maurer \& Macgill 2004; Volkov et al. 2005). Dessa forma, processos probabilísticos na colonização e extinção dos indivíduos nos habitats podem explicar as diferenças de composição e abundância das espécies nos platôs.

Como as espécies de plantas em uma comunidade aparecem e desaparecem aleatoriamente como resultado da imigração e extinção locais (Hubbell 2001), espera-se que a variação na abundância de uma determinada espécie apresente forte correlação espacial devido às limitações de dispersão. Como resultado, as comunidades de plantas na Amazônia são caracterizadas pelo grande número de espécies e elevada proporção de espécies raras (Ferreira et al. 2011).

A Teoria Neutra tende a explicar bem os padrões de composição de organismos residentes como plantas, pois estas apresentam maiores restrições quanto à dispersão, o que torna a dispersão um fator mais importante na composição das comunidades (Hubbell 1999; Chave 2004).

A alta diversidade encontrada nas parcelas analisadas nos seis platôs neste estudo demonstra o esperado para as florestas ombrófilas densas não alagáveis na Amazônia, onde o índice de diversidade (Shannon) varia de 3,83 a 5,85 

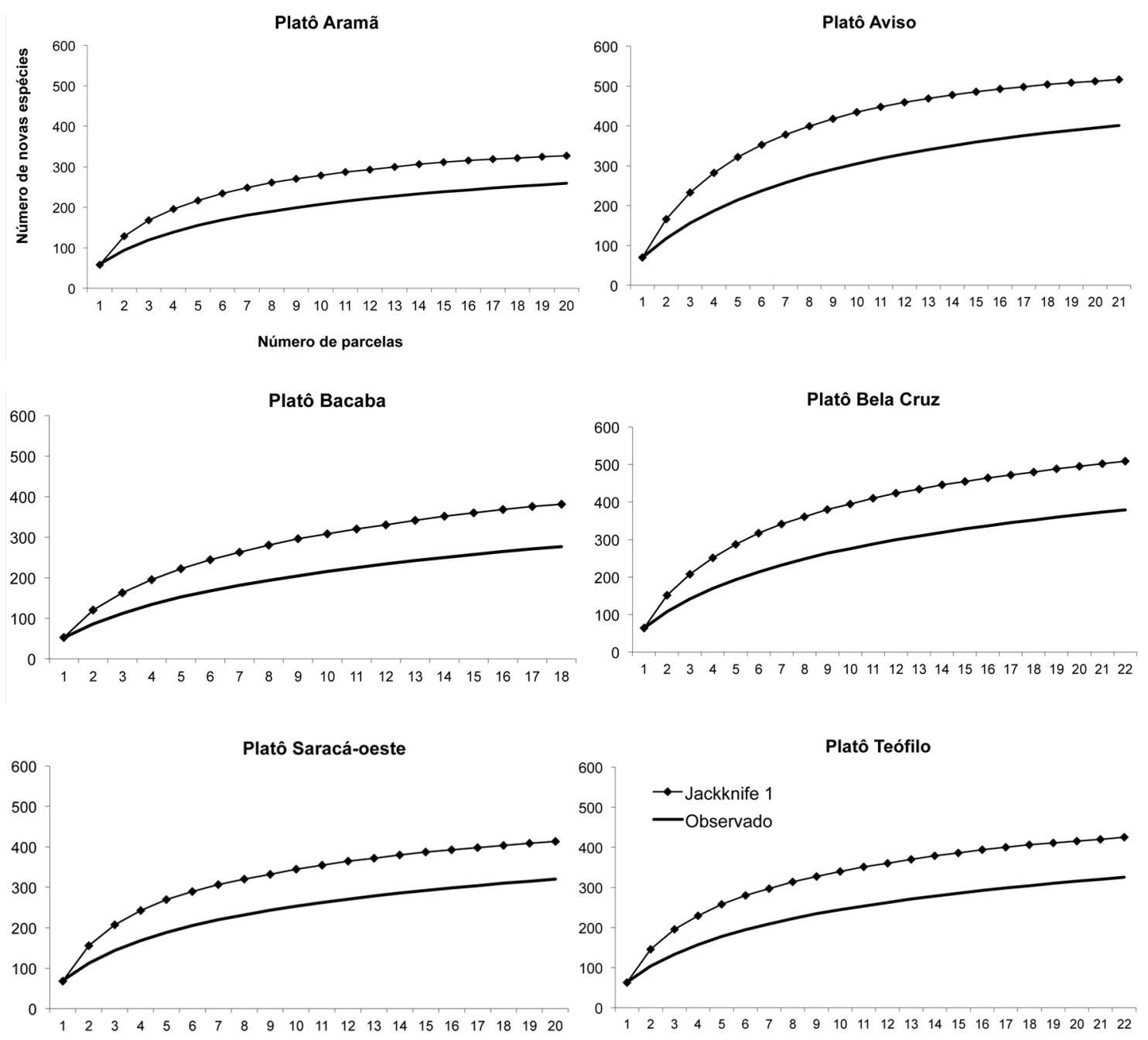

Figura 3 - Curva cumulativa de novas espécies (valor observado) e curvas do estimador de riqueza (Jackknife 1) nas parcelas avaliadas.

Figure 3 - The species-accumulation curve (observed value) and curves of the estimator of richness (Jackknife 1) in the plots evaluated.

(Campbell et al. 1986; Oliveira et al. 2008). Os resultados deste estudo confirmam a alta riqueza e diversidade de espécies em escala local (cada platô) e regional (todos os platôs).

Neste estudo não houve correlação significativa entre riqueza e distância nos platôs e entre platôs, com exceção do platô Aramã em que a riqueza foi negativamente correlacionada com a distância entre parcelas.

Apesar da variação de alguns fatores abióticos ser relativamente pequena nos platôs, variações micro-ambientais no espaço que caracterizam habitats pontuais, tais como, a distribuição de água no solo, incidência de luz, fertilidade, clareiras, topografia dentre outros, não analisados neste estudo, podem ser variáveis que estariam interferindo na variação da riqueza de espécies no platô Aramã. Assim, as espécies de plantas podem estar percebendo a heterogeneidade espacial em termos de seleção de micro habitats, estando também a variação de riqueza relacionada ao nicho ocupado.

Tuomisto et al. (2003) relatam que tanto as plantas como o ambiente pode ser estruturado espacialmente. A variação espacial causada pelas variáveis ambientais é aleatória, porém, autocorrelacionada, explicando porque locais próximos são mais similares em condições ambientais do que aqueles mais distantes. Além 

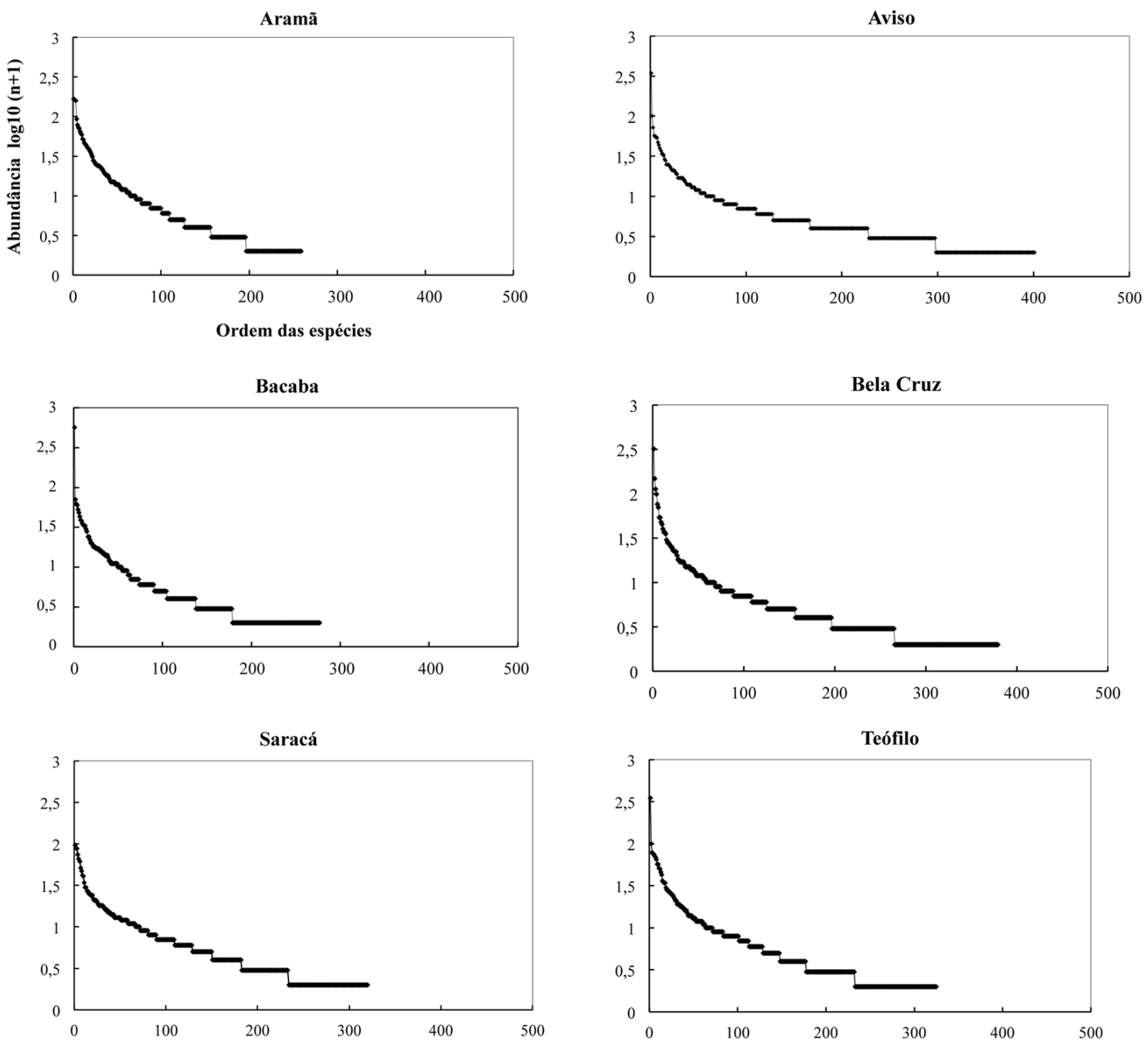

Figura 4-Diagrama de dominância mostrando a distribuição de abundância das espécies arbóreas nos seis platôs analisados. Figure 4 - Dominance diagram showing the distribution of tree species abundance in the six plateaus analyzed.

disso, diferentes espécies se especializam a diferentes porções do gradiente ambiental (Wilson 2000).

Vários estudos demonstram que muitas espécies de plantas tropicais são distribuídas de acordo com as variações das condições ambientais em várias escalas (Costa et al. 2005; Jones et al. 2006; Zumquim 2007).

A similaridade de espécies pode diminuir com a distância também devido ao efeito compartilhado de limitações na dispersão e autocorrelação espacial das características ambientais (Bell 2001).

Chust et al. (2006) estudaram espécies de árvores em uma floresta tropical no Panamá e observaram que $22 \%$ da variação florística foi explicada pela distância, $12 \%$ foi explicado exclusivamente pelas variáveis ambientais e $16 \%$ foi compartilhado, sendo que a maior parte da variação (49\%) permaneceu não explicada.

Estudos já foram feitos no intuito de compreender os efeitos da limitação de dispersão na composição de espécies nos trópicos (Condit et al. 2002). No entanto, a maioria da variação na composição de espécies em florestas tropicais não é explicada somente pela distância ou pelo ambiente (Duivenvoorden et al. 2002). Dessa forma, há necessidade de mais estudos antes de compreendermos os eventos que determinam a distribuição e a riqueza de espécies em florestas tropicais.

A variação na composição de espécies entre os seis platôs está relacionada à influência da diversidade beta, que é uma medida de dissimilaridade biológica 


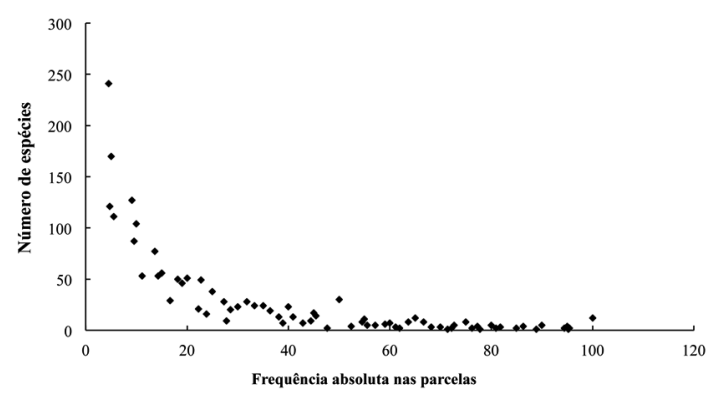

Figura 5 - Freqüência absoluta das espécies nas 123 parcelas analisadas neste estudo.

Figure 5 - Absolute frequency of species in 123 plots examined in this study.

entre ambientes e podem resultar da ação, em maior ou menor grau, de fatores ambientais, processos históricos e relações espaciais (Legendre et al. 2008).

Muitos estudos mostram a importância da dispersão e de fatores ambientais na variação de riqueza e composição de espécies em florestas tropicais, porém esses fatores variam de região para região e de acordo com escalas diferentes dentro de uma mesma região (Potts et al. 2002). Determinar a influência desses fatores na riqueza e composição das comunidades de plantas tem importante aplicação para a conservação (Pearman \& Weber 2007).

A manutenção da diversidade em várias escalas é muito debatida e diversas hipóteses já foram propostas (Janzen 1970; Hubbell 2001; Uriarte \& Reeve 2003; Tilmam 2004). A maioria das estratégias de conservação da biodiversidade em regiões tropicais é limitada devido ao reduzido nível de conhecimento sobre a distribuição das espécies.

O planejamento de estratégias de conservação na FLONA Saracá-Taquera deve considerar não somente a riqueza e diversidade locais de espécies, mas principalmente a conservação das espécies raras, pois, sem isto, corre-se o risco da eliminação da variação de espécies em escala regional.

Uma estratégia para a conservação da flora dos platôs na Flona Sacará-Taquera é ter vários platôs com tamanhos variados, mas com flora complementar, do que preservar somente um platô e perder a variação da composição de espécies de outros platôs. Por exemplo, na FLONA Sacará-Taquera, no platô Aviso, as porções leste e oeste foram deixados intactos. Esta pode ser uma estratégia importante para garantir a conservação dos platôs.

Outra alternativa seria preservar alguns platôs da Flona Sacará-Taquera, os que estiverem mais distantes entre si e que ainda não foram explorados, já que platôs mais distantes são mais distintos entre si em relação à composição de espécies como mostrado neste estudo.

\section{Agradecimentos}

À Universidade Federal Rural da Amazônia e ao Museu Paraense Emílio Goeldi. À Mineração Rio do Norte/MRN, em especial ao Ademar Cavalcanti Silva Filho, ao Instituto Chico Mendes de Biodiversidade (ICMBio). À Secretaria de Estado de Desenvolvimento, Ciência e Tecnologia - SEDECT e FAPESPA, a disponibilização de bolsa de mestrado em dois anos de curso.

\section{Referências}

Bell, G. 2001. Neutral macroecology. Science 293:24132418.

Campbell, D.D.; Daly, D.C.; Prance, G.T. \& Maciel, U.N. 1986. Quantitative ecological inventory of terra firme and varzea tropical forest on the rio Xingu, Brazilian, Amazon. Brittonia 38:369-393.

Carneiro, J.S. \& Valeriano, D.M. 2003. Padrão espacial da diversidade beta da Mata Atlântica - uma análise da distribuição da biodiversidade em um banco de dados geográficos. Anais do Simpósio Brasileiro de Sensoriamento Remoto 11: 629-636.

Chave, J. 2004. Neutral theory and community ecology. Ecology Letters 7: 241-253.

Chust, G.; Chave, J.; Condit, R.; Aguilar, S.; Lao, S. \& Pérez, R. 2006. Determinants and spatial modeling of tree beta - diversity in a tropical forest landscape in Panama. Journal of Vegetable Science 17: 83-92.

Cientec - Consultoria e Desenvolvimento de Sistemas. 2006. Mata nativa 2: Manual do usuário. Cientec, Viçosa. 295p.

Colwell, R.K.; Coddington, J.A. 1996. Estimating terrestrial biodiversity through extrapolation. In: Biodiversity: Measurement and evaluation. D. L. Hawksworth, London. Pp. 101-118

Colwell, R.K. 1997. EstimateS: Statistical Estimation of Species Richness and Shared Species from Samples. Version 8. User's guide and application published. Disponível em <http://viceroy.eeb.uconn. edu/ estimates>. Acesso em 20 Jan 2009.

Condit, R.; Pitman, N.; Leight Jr.E.G.; Chave, J.; Terborgh, J.; Foster, R.B.; Núñez, P.; Aguilar, S.; Valencia, R.; Villa, G.; Muller-Landau, H.C.; Losos, E. \& Hubbell, S.P. 2002. Beta diversity in tropical trees. Science 295: 666-668.

Condit, R.; Ashton, P.; Bunyavejchewin, S.; Dattaraja, H.S.; Davies, S.; Esufali, S.; Ewango, C.; Foster, R.; Gunatilleke, I.A.U.N.; Gunatilleke, C.V.S.; Hall, P.; Harms, Kyle E.; Hart, T.; Hernandez, C.; Hubbell, S.; Itoh, A.; Kiratiprayoon, S.; LaFrankie, J.; de Lao, Suzanne L.; Makana, Jean-Remy; Noor, Md.N.S.; 
Kassim, A.R.; Russo, S.; Sukumar, R.; Samper, C.; Suresh, H.S.; Tan, S.; Thomas, S.; Valencia, R.; Vallejo, M.; Villa, G. \& Zillio, T. 2006. The importance of demographic niches to tree diversity. Science 313 : 98-101.

Costa, F.R.C.; Magnusson, W.E. \& Luizão, R.C. 2005. Mesoscale distribution patterns of Amazonian understorey herbs in relation to topography, soil and watersheds. Journal of Ecology 93: 863-878.

Davidar, P.; Rajagopal, B.; Mohandass, D.; Puyravaud, J-P.; Condit, R.; Wright, S.J. \& Leigh JR, E.G. 2007. The effect of climatic gradients, topographic variation and species traits on the beta diversity of rain forests trees. Global Ecology and Biogeography 16: $510-518$

Duivenvoorden, J.F.; Svenning, J.C. \& Wright, S.J. 2002. Beta Diversity in Tropical Forests. Science 295: 636-637.

Esri - Environmental Systems Research Insitute. 2006. Arc View User's Guide. Redlands, ESRI, California. $187 \mathrm{p}$.

Ferreira, L.V.; Salomão, R.P.; Matos, D.C.L.; Pereira, J.L.V. 2011. Similaridade de espécies arbóreas em função da distância em uma floresta ombrófila na Floresta Nacional de Saracá-Taquera, Pará. Boletim do Museu Paraense Emílio Goeldi Ciências Naturais. 6: 295-306.

Giacomini, H.C. 2007. Os mecanismos de coexistência de espécies como vistos pela teoria ecológica. Oecologia Brasiliensis 11: 521-543.

Hubbell, S.P. 1999. Light-gap disturbances, recruitment limitation, and tree diversity in a neotropical forest. Science 283: 554-557.

Hubbell, S.P. 2001. The united neutral theory of biodiversity and biogeography. University Press, Princeton. 396p.

Hubbell, S.P. 2006. Neutral theory and the evolution of ecological equivalence. Ecology 87: 1397-1398.

Janzen, D.H. 1970. Herbivores and the number of tree species in tropical forest. American Naturalist 104: 501-528.

Jones, M.M.; Tuomisto, H.; Clark, D.B. \& Olivas, P. 2006. Effects of mesoscale environmental and dispersal limitation on floristic variation in rain forest ferns. Journal of Ecology 94: 181-195.

Kalkhoven, J.T.R. 1993. Survival of populations and the scale of fragmented agricultural landscape. In: Bunce, R.G.H.; Ryszkowski, L.; Paoletti, M.G. (Eds). Landscape Ecology and Agroecosystems. Lewis Publishers. 241p.

Kneitel, J.M. \& Chase, J.M. 2004. Trade-offs in community ecology: linking spatial scales and species coexistence. Ecology Letters 7: 69-80.

Krebs, C.J. 1999. Ecological methodology. AddisonWesley Educational Publishers, Menlo Park. 620p.

Legendre, P. 2008. Studying beta diversity: ecological variation partitioning by multiple regression and canonical analysis. Journal of Plant Ecology 1: 3-8.
Macarthur, R.H. \& Levins, R. 1964. Competition, habitat selection and character displacement in a partchy environment. Proceedings of National Academy of Sciences 51: 1207-1210.

Magurran, A.E. 1988. Ecological diversity and measurement. New Fatter Lane, London. 179p.

Magurran, A.E. 2004. Measuring biological diversity. Blackwell Publishing, Oxford. 256p.

Manly, B.F.J. Multivariate statistical methods: a primer. Chapman and Hall, London. 215p.

Matos, D.C.L. 2009. Estrutura e composição florística de comunidades de plantas em relação à distância geográfica na Amazônia Oriental. Dissertação de Mestrado. Universidade Federal Rural da Amazônia/ Museu Paraense Emílio Goeldi, Belém. 55p.

Mccune, B.; Mefford, M.J. 1999. PC-ORD version 4.0, multivariate analysis of ecological data, Users guide. Glaneden Beach, MjM Sofware Design, Oregon. 237p.

Maurer, B.A. \& Mcgill, B.J. 2005. Neutral and nonneutral macroecology. Basic and Applied Ecology 5: 413-422.

Mazancourt, C. 2001. Consequences of community drift. Science 293: 1772.

Mikkelson, G.M. 2005. Niche-based vs. neutral models of ecological communities. Biology and Philosophy 20: $557-566$.

Nekola, J.C. \& White, P.S. 1999. The distance decay in similarity in biogeography and ecology. Journal of Biogeography 26: 867-878.

Oliveira, A.N. de; Amaral, I.L.; Ramos, M.B.P.; Nobre, A.D.; Couto, L.B. \& Sahdo, R.M. 2008. Composição e diversidade florístico-estrutural de um hectare de floresta densa de terra firme na Amazônia Central, Amazonas, Brasil. Acta Amazônica 38: 627-642.

Pearman, P.B. \& Weber, D. 2007. Common species determine richness patterns in biodiversity indicator taxa. Biological conservation 138: 109-119.

Phillips, O.L.; Hall, P.; Gentry, A.H.; Sawyer, S.A. \& Vásquez, R. 1994. Dinamics and species richness of tropical rain forests. Proceedings of the National Academy of Sciences of the USA 91: 2805-2809.

Potts, M.D.; Ashton, P.S.; Kaufman, L.S. \& Plotkin, J.B. 2002. Habitat patterns in tropical rain forests: a comparison of 105 plots in northwest Borneo. Ecology 83: 2782-2797.

Pyke, C.R.; Condit, R.; Aguilar, S. \& Lao, S. 2001. Floristic composition across a climatic gradient in a Neotropical Lowland Forest. Journal of vegetation science 12: 553-566.

Radambrasil, Projeto. 1976. Folha SA.21 - Santarém, 10: 310-414.

Ruokolainen, K. \& Tuomisto, H. 2002. Beta-Diversity in Tropical Trees. Science 97: 1439 ${ }^{\mathrm{a}}$.

Salomão, R.P.; Rosa, N.A. \& Morais, K.A.C. 2007. Dinâmica da regeneração natural de árvores em áreas 
mineradas na Amazônia. Boletim do Museu Paraense Emílio Goeldi. Ciências Naturais 2: 85-139.

Scudeller, V.V.; Martins, F.R. \& Shepherd, G.J. 2001. Distribution and abundance of arboreal species in the atlantic ombrophilous dense forest in Southeastern Brazil. Plant ecology 152: 185-199.

Tilmam, D. 2004. Niche tradeoffs, neutrality, and community structure: a stochastic theory of resource competition, invasion, and community assembly. PNAS 101: 10854-10861.

Terborgh, J. \& Andersen, E. 1998. The composition of Amazonian florests: patterns at local e regional scales. Journal of Tropical Ecology 14: 645-664.

Ter Steege, H.; Sabatier, D.; Castellanos, H.; Van Andel, T.; Duivenvoorden, J.; de Oliveira, A.A.; Ek, R.; Lilwah, R.; Maas, P. \& Mori, S. 2000. An analysis of the floristic composition and diversity of Amazonian forests including those of the Guiana shield. Journal of Tropical Ecology 16: 801-828.

Ter Steege, H.; Pitman, N.; Sabatier, D.; Castellanos, H.; Hout, P.V.D.; Daly, D.C.; Silveira, M.; Phillips, O.; Vasquez, R.; Andel, T.V.; Duivenvoorden, J.; Oliveira, A.A.; Ek, R.; Wah, R.L.; Thomas, R.; Van Essen, J.; Baider, C.; Maas, P.; Mori, S.; Terborgh, J.; Vargas, P.N.; Mogollo’N.H. \& Morawetz, W. 2002. A spatial model of tree diversity and tree density for the Amazon. Biodiversity and Conservation 12: 2255-2277.

Tuomisto, H.; Ruokolainen, K.; Kalliola, R.; Linna, A.; Dan joy, W. \& Rodriguez, Z. 1995. Dissecting Amazonian Biodiversity. Science 269: 63-66.
Tuomisto, H. \& Poulsen, A.D. 1996. Influence of edaphic specialization on pteridophyte distribution in neotropical rain forests. Journal of biogeography 23: 283-293.

Tuomisto, H.; Ruokolainen, K. \& Yli-Halla, M. 2003. Dispersal, environment, and floristic variation of Western Amazonian Forests. Science 299: 241-244.

Uriarte, M. \& Reeve, H.K. 2003. Matchmaking and species marriage: a game-theory model of community assembly. PNAS 100: 1787-1792.

Veloso, H.P.; Rangel Filho, A.L. \& Lima, J.C.A. 1991. Classificação da vegetação brasileira adaptada a um sistema universal. IBGE, Rio de Janeiro. 124p.

Volkov, I.; Banavar, J.R.; He, F.; Hubbell, S.P. \& Maritan, A. 2005. Density dependence explains tree species abundance and diversity in tropical forests. Nature 438: 658-651.

Wilson, S.D. 2000. Heterogeneity, diversity and scale in plants communities. In: Hutchings, M.J.; Jonh, E.A. \& Stewart, A.J.A. (eds.). The ecological consequences of environmental heterogeneity. $40^{\text {th }}$ Symposium of the British Ecological Society.Cambridge University Press. Pp. 53-70.

Whittaker, R.H. 1965. Dominance and diversity in land plants communities. Science 147: 250-260.

Wright, S.J. 2002. Plant diversity in tropical forests: a review of mechanisms of species coexistence. Oecologia 130: 1-14.

Zuquim, G.; Costa, F.R.C. \& Prado, J. 2007. Fatores que determinam a distribuição de espécies de pteridófitas da Amazônia Central. Revista Brasileira de Biociências 5: 360-362. 